\title{
Efficacy and safety of percutaneous computed tomography-guided high-dose-rate interstitial brachytherapy in treatment of oligometastatic lymph node metastases of retroperitoneal space
}

\author{
Constanze Heinze, MD', Jazan Omari, MD', Matthias Manig, MD', Peter Hass, MD², Marino Venerito, MD², \\ Robert Damm, MD', Tomasz Jargiełto, MD4, Jens Ricke, MD5, Maciej Powerski, MD', Maciej Pech, MD',6 \\ 'Department of Radiology and Nuclear Medicine, Otto-von-Guericke University, Magdeburg, Germany, 2Department of Radiation Oncology. \\ Otto-von-Guericke University. Magdeburg, Germany, ${ }^{3}$ Department of Gastroenterology, Hepatology and Infectious Diseases, Otto-von- \\ Guericke University, Magdeburg, Germany, ${ }^{4}$ Department of Interventional Radiology and Neuroradiology, Medical University of Lublin, \\ Lublin, Poland, ${ }^{5}$ Department of Radiology, University Hospital Munich, Munich, Germany, ${ }^{2} 2^{\text {nd }}$ Department of Radiology. Medical University \\ of Gdansk, Gdansk, Poland
}

\begin{abstract}
Purpose: To assess efficacy, safety, and outcome of computed tomography (CT)-guided high-dose-rate (HDR) interstitial brachytherapy in patients with oligometastatic lymph node metastases of the retroperitoneal space.

Material and methods: 24 patients with a total of 47 retroperitoneal lymph node metastases from different primary tumors were treated with CT-guided interstitial brachytherapy using an ${ }^{192}$ Ir source (single fraction irradiation). Every three months after treatment, clinical and imaging follow-up were conducted to evaluate local control and safety.

Results: Median follow-up was 9.6 months (range, 2.9-39.0 months). Local tumor control rate was 95.7\%. The median diameter of the gross tumor volume was $2.2 \mathrm{~cm}$ (range, $1-8.6 \mathrm{~cm}$ ), treated with a median $\mathrm{D}_{100}$ (minimal enclosing tumor dose) of 14.9 Gy (range, 4.5-20.6 Gy). One severe adverse event (grade three) was recorded.

Cumulative median progression-free survival was 4.2 months (range, 1.4-23.7 months), and cumulative median overall survival after interstitial brachytherapy was 15.9 months (range, 3.8-39.0 months).

Conclusions: CT-guided HDR interstitial brachytherapy is a safe and feasible method for local ablation of oligometastatic lymph node metastases of the retroperitoneal space, and might provide a well-tolerated additional therapeutic option in the multidisciplinary management of selected patients.

Key words: lymph node metastases, image-guided intervention, radiation therapy/oncology, interstitial brachytherapy.

\section{Purpose}

Almost all types of solid cancer have the potential of lymphatic dissemination to the retroperitoneal space, especially squamous cell carcinoma of the pelvis, urinary or gynecological system as well as gastrointestinal adenocarcinoma or renal cell carcinoma [1]. Therapeutic options depend on the type of primary tumor, location, and number/volume of the retroperitoneal lymph node metastases (rLNM), and on the patient's performance status. However, little is known about the impact of the oligometastatic state in these patients. The term 'oligometastases' was coined by Hellmann et al. in 1995; the authors hy- pothesized that the process of cancer metastases develop along a continuum from locally limited to polymetastatic disease and therefore, over time, malignant cells acquire widespread metastatic potential [2]. Furthermore, they suggested that selected patients with a restricted metastatic capacity could be classified into a specific intermediate transitional stage between single metastasis and polymetastatic disease. Nowadays, precise clinical definitions vary among publications. In general, oligometastatic disease is defined by metastases that are limited in number (typically less than five) and location, but more importantly are amendable for regional treatment, aim-

Address for correspondence: Constanze Heinze, MD, Department of Radiology and Nuclear Medicine, $\quad$ Received: 25.01 .2019 Otto-von-Guericke University, Leipziger Strasse 44, 39120 Magdeburg, Germany, phone: +49391 6713030, Accepted: 28.08 .2019 fax: + 49391 6713029, $\bowtie$ e-mail: constanze.heinze@med.ovgu.de

Published: 30.10 .2019 
ing for a complete resection/ablation [3,4,5]. In general, metastatic malignancies are associated with a poor prognosis and (according to the current standard of care) primarily treated with systemic chemotherapy or molecular targeted therapy. However, in the described model, local tumor control would have a potential to decelerate cancer progression or yield systemic control. Furthermore evidence exists that patients with oligometastases can even be cured by resection of the lesions, i.e. most frequently reported in patients with colorectal carcinoma after resection of liver metastases [6,7].

However, retroperitoneal lymph node dissection is a surgical challenge, especially in a post-chemotherapeutic setting with significant morbidity [8]. Alternative less invasive local treatments include stereotactic body radiation (SBRT), radiofrequency ablation (RFA), and image-guided high-dose-rate interstitial brachytherapy $(\mathrm{HDR}-\mathrm{iBT}=\mathrm{iBT})$. Out of the toolbox of local ablation techniques, $\mathrm{iBT}$ is a relatively new technique, where an iridium $192\left({ }^{192} \mathrm{Ir}\right)$ source is temporarily introduced into the metastatic lesions via percutaneously implanted applicators, which are placed under imaging guidance in a minimal invasive intervention; therefore, enabling a clearly delineated single fraction irradiation of the target volume. IBT has already been shown to be a gentle treatment with a minimum of complications in ablation of primary or secondary malignancies at various sites, e.g. colorectal cancer or hepatocellular carcinoma $[9,10,11,12]$. To our knowledge, no data has been published so far evaluating the efficacy of iBT in the treatment of rLNM. In this study, we retrospectively analyzed safety and efficacy in a cohort of 24 patients with 47 rLNM who underwent CT-guided iBT.

\section{Material and methods}

\section{Eligibility criteria and patients}

Patient recruitment was carried out between March 2015 and March 2017. We retrospectively included 24 patients ( 13 male and 11 female; median age, 55.5 years; range, 29-81 years) with 47 rLNM. All patients were diagnosed with different types of primary tumors, all histologically proven (for detailed patients characteristics see Table 1).

All patients displayed tumor progression at the time of referral to our clinic and every case was discussed in an interdisciplinary tumor board. Furthermore, inclusion criteria were as follow: a) Technically unresectable metastases, assessed by a surgeon with expertise in the field of visceral surgery; b) Medical contraindication for resection or severe comorbidities; c) Refusal of surgery; d) Patients considered unfit for chemotherapy or lack of chemotherapy options; e) Refusal of chemotherapy; f) The East Coast Oncology Group (ECOG) performance status below two; g) Platelet count $>50,000 / \mathrm{nl}$, international normalized ratio $($ INR $)<1.5$, partial thromboplastin time $<50$ seconds. No upper limit concerning maximum tumor diameter or number of lesions was placed; however, all lesions had to be amenable for regional treatment aiming for a complete ablation. Contraindications for local ablation included: a) Peritoneal carcinomatosis; b) Prognosis limiting, widespread systemic disease; c) Lack of consent.

Prior to ablation, 17 out of 24 patients were treated with systemic treatment including immunotherapy. Sev-

Table 1. Patients characteristics

\section{Variables}

\begin{tabular}{lc}
\hline Total number of patients $(n)$ & 24 \\
\hline Patient sex & 13 \\
\hline Men & 11 \\
\hline Women & \\
\hline Age at time of diagnosis (years) & 55.5 \\
\hline Median & $29-81$ \\
\hline Range
\end{tabular}

Primary tumor entity: number of patients/total number of lesions per entity $(n)$

\begin{tabular}{ll}
\hline Colorectal carcinoma & $5 / 7$ \\
\hline Cholangiocellular carcinoma & $4 / 7$ \\
\hline
\end{tabular}

Renal cell carcinoma $\quad 3 / 7$

Pancreatic ductal adenocarcinoma $\quad 3 / 5$

Hepatocellular carcinoma 2/4

Ovarian cancer 2/2

Cancer of unknown primary 2/5

Malignant pheochromocytoma $\quad 1 / 4$

Malign melanoma $1 / 3$

Urothelial carcinoma $\quad 1 / 3$

Distant metastasizing

\begin{tabular}{lc}
\hline Synchronous & 9 \\
\hline Metachronous & 15 \\
\hline
\end{tabular}

Number of lesions $(n) \quad 47$

\begin{tabular}{lc}
\hline Maximum diameter $(\mathrm{cm})$ & \\
\hline Median & 2.2 \\
\hline Range & $1.0-8.6$
\end{tabular}

Lesions per patient ( $n$ )

Median 2

Range 1-4

Administered $\mathrm{D}_{100}$; range (Gy)

\begin{tabular}{lc}
\hline Median & 14.9 \\
\hline Range & $4.5-20.6$
\end{tabular}

Follow-up time (months)

\begin{tabular}{lc}
\hline Median & 9.6 \\
\hline Range & $2.9-39.0$
\end{tabular}


en patients did not receive any chemotherapy due to reduced general condition, comorbidities, or refusal of systemic treatment. All, except two patients, underwent resection of the primary tumor or metastatic lesions, including local ablation using iBT, RFA, or microwave ablation (MWA) of liver, lung, or lymph nodes.

Prior to iBT of rLNM, all patients underwent a full clinical status evaluation with a physical examination and laboratory assessment. Additionally, a whole-body contrast-enhanced CT was performed to obtain a complete staging. A positive vote of the ethics committee for the analysis of the patient data was received, and informed consent was obtained from all individual participants included in the study.

\section{Interventional technique and irradiation}

The technique has been described elsewhere in detail $[10,13,14]$. In short, under guidance of a fluoroscopy-CT (Toshiba, Aquilion, Japan), an 18-gauge needle was introduced into the target lesions and a flexible $6 \mathrm{~F}$ catheter sheath (Radifocus, Terumo ${ }^{\mathrm{TM}}$ Introducer II, Terumo Europe, Leuven, Belgium) was inserted over a stiff angiography guide wire using Seldinger's technique. Thereafter, the angiographic guidewire was removed and replaced by a $6 \mathrm{~F}$ afterloading catheter (Afterloadingkatheter, Primed ${ }^{\circledR}$ Medizintechnik GmbH, Halberstadt, Germany). The interventional procedure was performed under local anesthesia (lidocaine) and analgosedation (midazolam and fentanyl). The number and arrangement of the implanted catheters was determined by the shape, size, and location of the target lesion. After catheter positioning, a contrast-enhanced CT scan in breath-holding technique was obtained to document correct catheter positioning and to plan irradiation. On these images, the target volume was drawn precisely as gross tumor volume (GTV) and clinical target volume (CTV); additionally, organs at risk (OARs) such as duodenum or spinal cord were outlined by an interventional radiologist and radiation oncologist. The ends of the afterloading catheters were secured to the skin with a suture and the tip of the catheter was presumably in a fixed position; therefore, planning target volume (PTV) and CTV are in accordance with one another. Dose calculation was performed using the acquired dataset with Oncentra-Masterplan (Oncentra ${ }^{\circledR}$ Brachy treatment planning system, Elekta AB, Stockholm, Sweden) and the calculated isodose lines, relative to the CTV, were controlled and adapted slice by slice. All irradiations were administered as single fraction irradiations using an ${ }^{192}$ Ir source with a nominal activity of $10 \mathrm{Ci}$. Depending on the histological type of the primary tumor, a reference dose of 15-20 Gy was prescribed to our patients (e.g. 15 Gy for renal cell carcinoma and hepatocellular carcinoma, 20 Gy for cholangiocellular carcinoma, malignant pheochromocytoma). The reference dose was defined as the minimum dose enclosing the complete CTV $\left(D_{100}\right)$. There was no limitation regarding higher doses inside the tumor volume. However, depending on adjacent OARs, dose limitations were taken into account, i.e. gastric mucosa $(<15.5 \mathrm{~Gy} / \mathrm{ml})$ [15]. After irradiation, the catheters were removed and the puncture channels were sealed using thrombogenic material $\left(\right.$ Gelfoam $^{\circledR}$; Pfizer Inc., New York, US). Figure 1 illustrates the interventional technique.

\section{Follow-up}

Every three months after iBT, clinical laboratory and imaging follow-up (contrast-enhanced whole-body CT) were performed. Local tumor control (LTC) and progression-free survival (PFS) were assessed by employing RECIST criteria (RECIST version 1.1). LTC was defined as decreasing or stable presentation of the target lesion after iBT. PFS was defined as LTC without tumor progression at any other side after iBT. Overall survival (OS) was calculated from the date of ablation to death. Adverse events were defined according to the Common Terminology Criteria for Adverse Events (CTCAE version 4.03).

\section{Study design and statistical analysis}

The analyzed data was retrospectively collected from our internal database ASENA ${ }^{\circledR}$ (LoeScap Technology $\mathrm{GmbH}$ ). Primary endpoints were LTC and safety; secondary endpoints were OS and PFS. The results were analyzed in a non-randomized and retrospective approach. LTC, OS, and PFS were evaluated employing the Kaplan-Meier method with SPSS (IBM Corp. Released 2013. IBM SPSS Statistics for Windows, Version 22.0. Armonk, NY: IBM Corp). Safety was evaluated descriptively.

\section{Results}

Median diameter of the target lesion was $2.2 \mathrm{~cm}$ (range, $1.0-8.6 \mathrm{~cm}$ ) treated with a median $\mathrm{D}_{100}$ of $14.9 \mathrm{~Gy}$ (range, 4.5-20.6 Gy). All 47 LNM were located in the retroperitoneal space at/below the coeliac trunk and superior to the aortic bifurcation in a paraaortic, paracaval, or interaortocaval distribution. In case of irradiation in the vicinity of an OAR, the intended minimum tumor dose $\left(D_{100}\right)$ had to be lowered; therefore, eight out of 47 lesions received less than $10 \mathrm{~Gy}$. During the treatment, no OAR was irradiated in excess of the critical value. Twenty-one patients were treated in one session, three patients received two sessions due to progressive disease within the follow-up period. We treated a median of $2 \mathrm{rLNM}$ per patient (range, 1-4). A mean of 1.4 catheters (range, 1-4) was employed to achieve full coverage of the target lesion, and the mean irradiation time per CTV was $19.6 \mathrm{~min}$ (range, 4.1-55.3 $\mathrm{min}$ ).

Mean hospital stay was 4.7 days (range, 2-12 days). We report two cases of small pneumothoraces that regressed spontaneously (classified as severe adverse event grade 1). We report four other cases of mild or moderate adverse events: one mild allergic reaction (urticaria, itching) to metamizole that was administered post-interventional, it was treated successfully with dimetindene and cimetidine; two other patients had slight side effects like sickness and emesis, and a fourth patient showed asymptomatic hematuria that regressed spontaneously. In one patient with metastasized pancreatic ductal adenocarcinoma, a small post-interventional abscess with a concomitant spondylodiscitis occurred, initially requiring intra- 

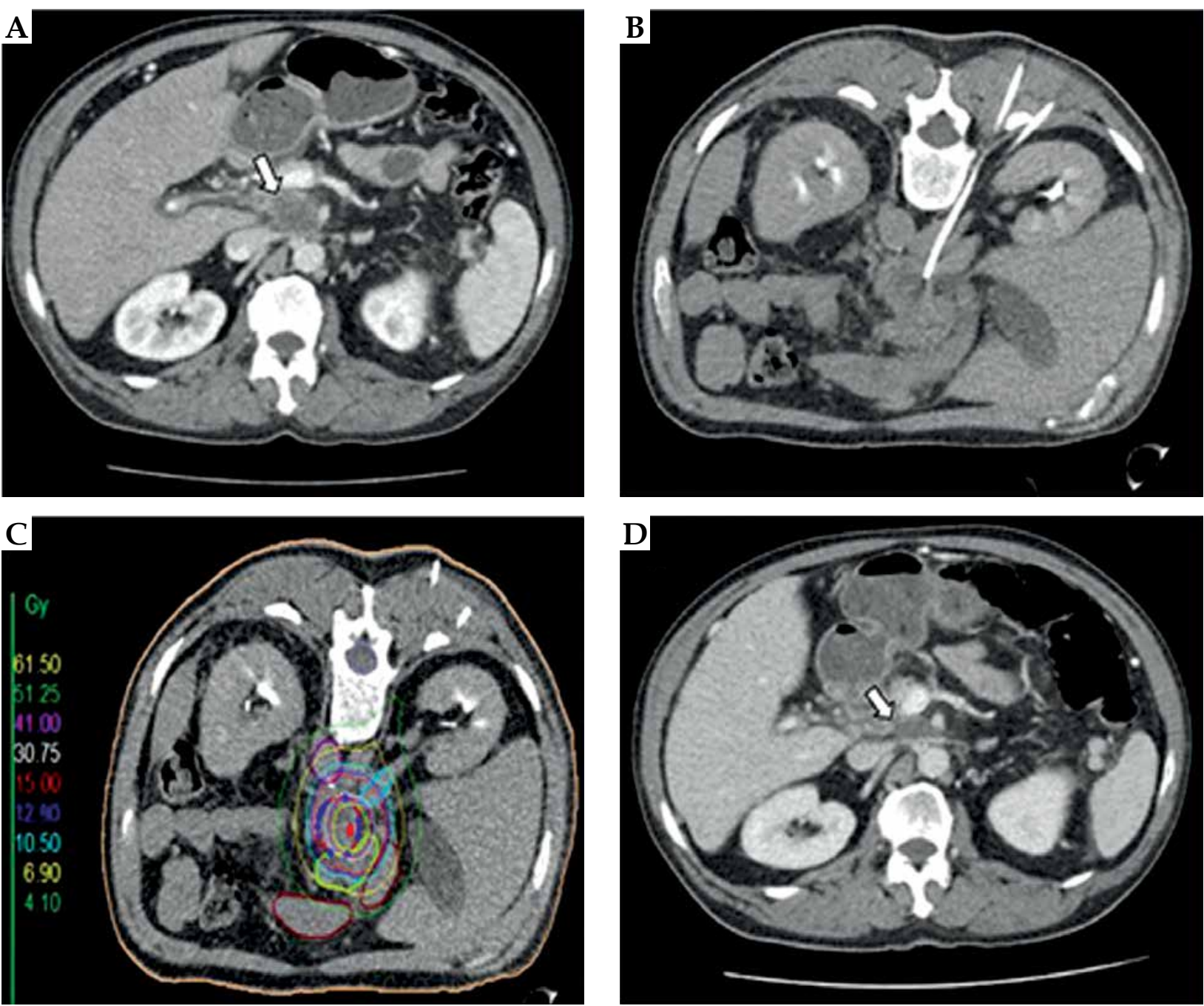

Fig. 1. Interventional technique and local tumor control in a patient with a retroperitoneal lymph node metastasis (rLNM) from pancreatic ductal adenocarcinoma. A) Pre-interventional contrast-enhanced CT slice showing a rLNM (white arrow) located below the coeliac trunk; B) Peri-interventional CT slice with one percutaneously implanted brachytherapy catheter. The patient is placed in the prone position; C) Planning CT with indicated clinical target volume (CTV; blue line), isodose lines, and marked organs at risk (e.g. gastric and duodenal structures). The color-coded isodose levels are shown in Gy (scale on the left side of the image); D) Contrast-enhanced CT slice three months after high-dose-rate interstitial brachytherapy showing partial remission of the treated lesion

venous antibiotics; although, seven months post-iBT, an operative short segment fixation was indicated to secure stability of the spine (classified as a severe adverse event grade 3 ).

During the median follow-up time of 9.6 months (range, 2.9-39.0 months), two patients exhibited local recurrence of GTV at eight months and four months after iBT, resulting in LTC rate of $95.7 \%$ in the Kaplan-Meier analysis, including lesions treated with less than $10 \mathrm{~Gy}$ (Figure 2). The two recurrent lesions were rLNM of colorectal cancer and a carcinoma of unknown primary (CUP), covered with $\mathrm{D}_{100}$ of $20.5 \mathrm{~Gy}$ and $4.5 \mathrm{~Gy}$ at time of treatment, respectively.

Progression-free survival ranged from 1.4-23.7 months, with a median of 4.2 months (Figure 3 ). Within the follow-up period, all patients showed systemic progressive disease. In the time between iBT and systemic progression, 10/24 patients received specific tumor therapy (i.e. chemo- and immunotherapy, one patient was treated with iBT of the liver). At time of censoring, 14 out of 24 patients $(58 \%)$ were still alive, resulting in a median OS of 15.9 months (range, 3.8-39.0 months) (Figure 4).

\section{Discussion}

Lymph node metastases are often the first site of metastases in a variety of tumors and, therefore, are critical for staging and prognosis [16]. Nevertheless, patients with a disseminated disease are, according to the current standard of care, in general, treated with adjuvant or palliative chemotherapy depending on the primary tumor and the patient's performance status. In several urological malignancies, a surgical approach is of prognostic and therapeutic value, for instance, in testicular cancer 


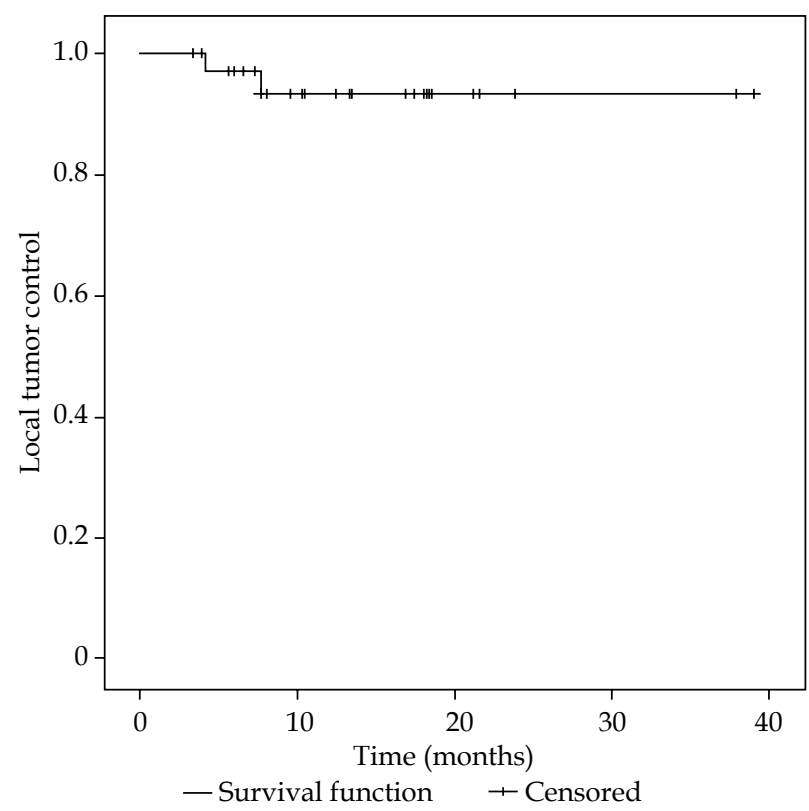

Fig. 2. Local tumor control after high-dose-rate interstitial brachytherapy

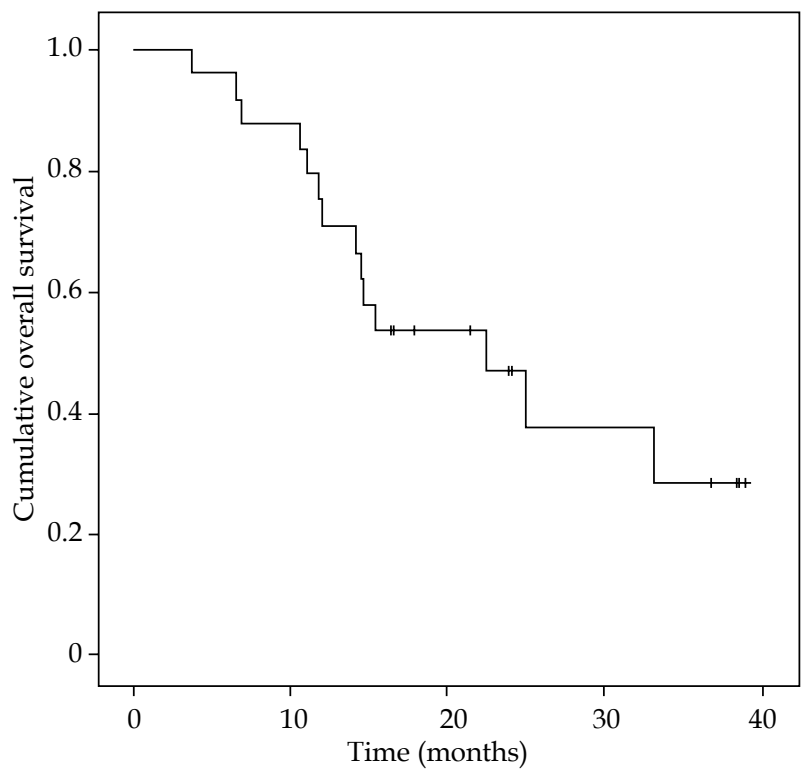

- Survival function $\quad+$ Censored

Fig. 4. Overall survival after high-dose-rate interstitial brachytherapy. At date of censoring, 14 out of 24 patients are still alive

with a reported overall complication rate of $20-35 \%$ and mortality of $1 \%[8,17]$. However, retroperitoneal lymph node dissection (RLND) remains controversial for other malignancies, for example in renal cell cancer, a randomized trial failed to show a survival advantage of a complete RLND in combination with a radical nephrectomy compared to radical nephrectomy alone [18]. Nevertheless, in numerous cases, resection might not be possible due to location or accessibility of the metastases, or due to contraindications for surgery or general anesthesia, apart from associated morbidity and mortality.

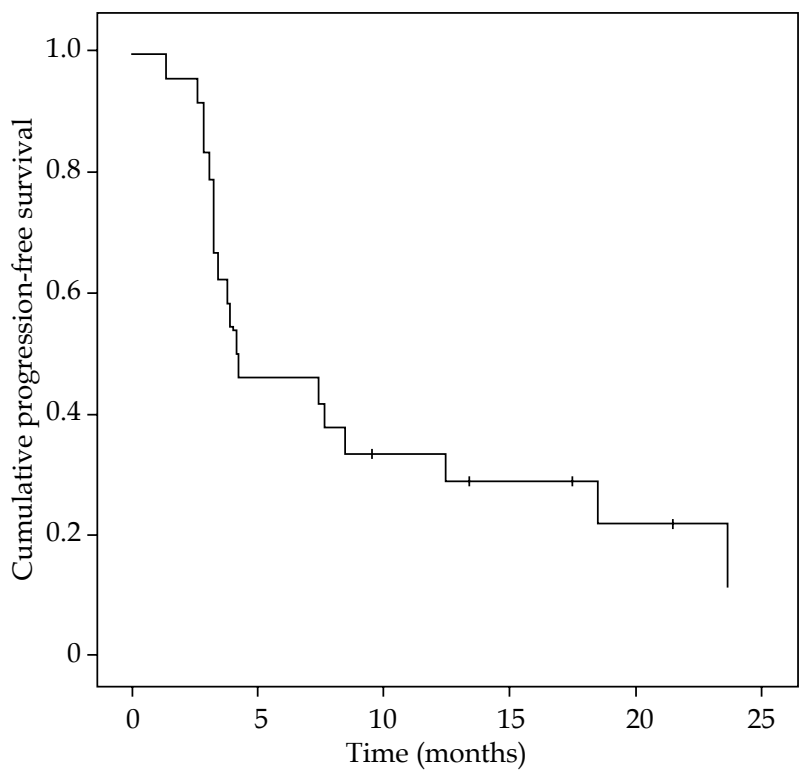

- Survival function + Censored

Fig. 3. Progression-free survival after high-dose-rate interstitial brachytherapy

Data regarding stereotactic body radiotherapy (SBRT) of rLNM are scarce. Bignardi et al. treated 19 patients with rLNM of various tumors with SBRT resulting in LTC of $77.8 \% \pm 13.9 \%$ (median \pm standard error) at both 12 and 24 months [19]. In one patient, a grade 3 adverse event occurred, but unclear whether associated with previous surgery or previous conformal radiotherapy. Nonetheless, according to the literature, acute and late toxicities after SBRT of intraperitoneal LNM vary between $14.1-34.0 \%$ and $0-11.6 \%$,with two grade 3 acute and one grade 4 late toxicity events reported in two studies $[20,21]$. Therefore, the question may arise: what is the benefit of iBT opposed to SBRT? SBRT permits precise but repeated irradiation. The delivery of an effective dose to the target is closely associated with significant exposure of surrounding tissue, resulting in restrictions with respect to size and number of lesions as well as location and, therefore, resulting in varying acute and late toxicities, e.g. for abdominal SBRT [22]. IBT allows one-time radiation with a high ablative dose inside the target volume preserving adjacent OAR from potentially harmful exposure as a result of favorable dosimetric characteristics. Thus, iBT demonstrates opportunities for the radiation of tumors in complex locations without concrete size limitations.

Furthermore, in order to enable the delivery of an effective dose in some cases, fiducial markers need to be implanted in or near the tumor before SBRT [23]. Therefore, SBRT can also be an invasive method associated with potential complications such as bleeding, especially if not performed by experienced radiation oncologists.

The applied ablative dose delivered via iBT and SBRT cannot be compared exactly. Recalculation using the linear-quadratic model identifies the equivalent biologically effective dose (eqBED), however, it represents an 
approximation. Although the eqBED appears lower for iBT inside the tumor, it theoretically increases exponentially towards the center and would be infinitely high at the point source; additional aspect to consider is the biological effects of one-time radiation compared to repeated radiotherapy [24]. Moreover, our results confirm that the applied dose was sufficient to achieve high local control rate.

Finally, further investigations need to be conducted to enlighten the differences and similarities of the two techniques and especially, to allow appropriate patient selection for both techniques according to the expected outcome.

To our knowledge, apart from case reports and case series $[25,26,27]$, little data exists on the efficacy and safety of percutaneous local ablative techniques including iBT, RFA, and microwave ablation in the treatment of rLNM. There are three studies investigating the feasibility of RFA in the ablation of rLNM. Machi et al. performed sonographic-guided RFA in seven patients (colorectal, renal, and prostate cancers), with LTC rate of $71.4 \%$ and severe complications such as enterovesical fistula and fecal incontinence [28]. In the study of Arellano et al., eight patients with gynecologic malignancies were enrolled, of which only five received treatment (two patients could not be treated due to proximity of adjacent heat-sensitive structures) and did not show any local recurrence or severe adverse events [29]. Gao et al. treated 19 patients with retroperitoneal metastasized hepatocellular carcinoma using CT-guided RFA, resulting in LTC rate after 10 months of $41.7 \%$ and OS after 1 year of $26.3 \%$, compared to a matched control group with 13 patients and a one-year OS of 7.7\% [30]. These findings emphasize the well-known technical limitations of this thermal technique, leading to a potential incomplete ablation and reduced LTC rates, i.e. a large tumor mass (maximal tumor diameter of $5 \mathrm{~cm}$ ) and major vessels close to the target volume inducing a potential cooling effect. Furthermore, severe adverse events can occur due to the vicinity to critical heat-sensitive organs, requiring a careful patient selection. In contrast, iBT is unrestricted of these limitations and furthermore, compared to surgical resection, offers not only advantages in terms of treatment tolerability but also accessibility of lesions (in number and location).

To our knowledge, no data regarding iBT of rLNM exists; however, Collettini et al. treated 10 patients with intraperitoneal LNM with iBT, and reported LTC rate of $80 \%$ during median follow-up of 13.2 months [31].

Our study provides superior LTC rate of $95.7 \%$ within median follow-up period of 9.6 months. Furthermore, in 24 patients and 47 lesions, we report one severe adverse event grade three. Although in this patient, an operative intervention was indicated, it should be emphasized that at the day of censoring, the patient is still alive and free of local recurrence or systemic progression after being diagnosed with metastasized pancreatic ductal adenocarcinoma in 2013.

Our findings of median PFS of 4.2 months (range, 1.4-23.7 months) and median OS of 15.9 months (range,
3.8-39.0 months) after iBT are not beneficial from an oncological perspective due to the heterogeneity and rather small cohort; therefore, these results are not comparable to the existing literature. However, at date of censoring, 14 out of 24 patients are still alive; moreover, we report two long-term survivors with 38.5 and 39.0 months after iBT diagnosed with renal cell carcinoma and a malignant pheochromocytoma, respectively.

In general, treatment of metastatic disease is challenging with an increasing tendency towards an individually tailored anticancer therapy to achieve the best possible outcomes. From an oncological perspective, the state of oligometastatic disease has been increasingly spotlighted in treatment strategies of metastatic disease, with the focus on colorectal cancer $[32,33]$. Therefore, one can argue that in an oligometastatic setting, the rationale for treating rLNM should be the same as for selected patients with liver or lung metastases, with the aim of complete ablation and therefore, the maximal possible reduction of the tumor cell biomass (i.e. cytoreduction) within the bounds of tolerable toxicity. Hence, a rational approach proposes that metastatic ablation can extend PFS, prolong pause of cytostatic treatment, or enable de-escalation to a maintenance therapy. Additionally, considering reported long-term survivors after local ablation or results of studies (e.g. Pan et al.) showing a favorable impact of local ablation of LNM of hepatocellular carcinoma on survival (RFA group compared to a non-RFA matched cohort showing median OS of 13.0 months vs. 7.9 months, respectively), confirms that the effect of local ablative techniques on survival is far from being answered [34].

Another important rationale for treating rLNM in rather palliative setting might be the precise ablation of metastases causing symptoms or the ablation of metastases at risk to cause complications in future, in order to decrease clinical symptoms by the reduction of tumor volume or to delay the time to clinical symptoms and therefore, improve the quality of life.

Severe limitations of our study need to be addressed including its retrospective nature and low number of cases. Also, the heterogeneity of the treated cohort with respect to primary tumor, disease stage, and previous treatment resulting in a cumulative PFS and OS that is not beneficial from an oncological perspective. Therefore, a prospective trial with a higher caseload limited to a distinct tumor entity might enlighten the oncological effect of iBT with respect to the primary tumor and the disease stage.

In spite of these limitations, our study demonstrates that iBT is not only a feasible alternative to SBRT or RLND with treatment and primary tumor independent effective LTC rates, but also offers a well-tolerated and safe therapeutic option in the multidisciplinary management of oligometastatic selected patients.

\section{Conclusions}

For patients presented with oligometastatic rLNM, iBT is a safe and particularly effective ablative technique that provides a promising treatment option. 


\section{Disclosure}

The authors report no conflict of interest.

\section{References}

1. Luo $\mathrm{CH}$, Zou B. Retroperitoneal lymph node metastases. Springer Netherlands, Dordrecht 2018; 273-276.

2. Hellman S, Weichselbaum RR. Oligometastases. J Clin Oncol 1995; 13: 8-10.

3. Weichselbaum RR, Hellman S. Oligometastases revisited. Nat Rev Clin Oncol 2011; 8: 378-382.

4. Reyes DK, Pienta KJ. The biology and treatment of oligometastatic cancer. Oncotarget 2015; 6: 8491-8524.

5. Kaneda H, Saito Y. Oligometastases: Defined by prognosis and evaluated by cure. Cancer Treatment Communications 2015; 3: 1-6.

6. Tomlinson JS, Jarnagin WR, DeMatteo RP et al. Actual 10-year survival after resection of colorectal liver metastases defines cure. J Clin Oncol 2007; 25: 4575-4580.

7. Rees M, Tekkis PP, Welsh FKS et al. Evaluation of long-term survival after hepatic resection for metastatic colorectal cancer: a multifactorial model of 929 patients. Ann Surg 2008; 247: 125-135.

8. Baniel J, Sella A. Complications of retroperitoneal lymph node dissection in testicular cancer: primary and post-chemotherapy. Semin Surg Oncol 1999; 17: 263-267.

9. Ricke J, Wust P, Stohlmann A et al. CT-guided interstitial brachytherapy of liver malignancies alone or in combination with thermal ablation: phase I-II results of a novel technique. Int J Radiat Oncol Biol Phys 2004; 58: 1496-1505.

10. Collettini F, Lutter A, Schnapauff D et al. Unresectable colorectal liver metastases: percutaneous ablation using CT-guided high-dose-rate brachytherapy (CT-HDBRT). Rofo 2014; 186: 606-612.

11. Mohnike K, Wieners G, Schwartz F et al. Computed tomography-guided high-dose-rate brachytherapy in hepatocellular carcinoma: safety, efficacy, and effect on survival. Int I Radiat Oncol Biol Phys 2010; 78: 172-179.

12. Heinze $C$, Omari J, Othmer $M$ et al. Image-guided interstitial brachytherapy in the management of metastasized anal squamous cell carcinoma. Anticancer Res 2018; 38: 5401-5407.

13. Bretschneider T, Ricke J, Gebauer B et al. Image-guided highdose-rate brachytherapy of malignancies in various inner organs - technique, indications, and perspectives. J Contemp Brachytherapy 2016; 8: 251-261.

14. Ricke J, Wust P. Computed tomography-guided brachytherapy for liver cancer. Semin Radiat Oncol 2011; 21: 287-293.

15. Streitparth F, Pech M, Böhmig M et al. In vivo assessment of the gastric mucosal tolerance dose after single fraction, small volume irradiation of liver malignancies by computed tomography-guided, high-dose-rate brachytherapy. Int J Radiat Oncol Biol Phys 2006; 65: 1479-1486.

16. Wittekind C. Diagnosis and staging of lymph node metastasis. Recent Results Cancer Res 2000; 157: 20-28.

17. Smith ZL, Werntz RP, Eggener SE. Testicular cancer: epidemiology, diagnosis, and management. Med Clin North Am 2018; 102: 251-264.

18. Blom JHM, van Poppel H, Maréchal JM et al. Radical nephrectomy with and without lymph-node dissection: final results of European Organization for Research and Treatment of Cancer (EORTC) randomized phase 3 trial 30881. Eur Urol 2009; 55: 28-34.

19. Bignardi M, Navarria P, Mancosu P et al. Clinical outcome of hypofractionated stereotactic radiotherapy for abdominal lymph node metastases. Int J Radiat Oncol Biol Phys 2011; 81: 831-838.
20. Jereczek-Fossa BA, Piperno G, Ronchi $S$ et al. Linac-based stereotactic body radiotherapy for oligometastatic patients with single abdominal lymph node recurrent cancer. Am J Clin Oncol 2014; 37: 227-233.

21. Franzese C, Cozzi L, Franceschini D et al. Role of stereotactic body radiation therapy with volumetric-modulated arcs and high-intensity photon beams for the treatment of abdomino-pelvic lymph-node metastases. Cancer Invest 2016; 34: 348-354.

22. Thomas TO, Hasan S, Small W, Jr. et al. The tolerance of gastrointestinal organs to stereotactic body radiation therapy: what do we know so far? J Gastrointest Oncol 2014; 5: 236-246.

23. Jereczek-Fossa BA, Ronchi S, Orecchia R. Is stereotactic body radiotherapy (SBRT) in lymph node oligometastatic patients feasible and effective? Rep Pract Oncol Radiother 2015; 20: $472-$ 483.

24. Vaiserman A, Koliada A, Zabuga O et al. Health impacts of low-dose ionizing radiation: current scientific debates and regulatory issues. Dose Response 2018; 16: 1559325818796331.

25. Tian G, Jiang TA. US-guided percutaneous laser ablation of refractory metastatic retroperitoneal lesions: A care-compliant case report. Medicine (Baltimore) 2017; 96: e6597.

26. Jiang TA, Deng Z, Tian G et al. Percutaneous laser ablation: a new contribution to unresectable high-risk metastatic retroperitoneal lesions? Oncotarget 2017; 8: 2413-2422.

27. Gervais DA, Arellano RS, Mueller PR. Percutaneous radiofrequency ablation of nodal metastases. Cardiovasc Intervent Radiol 2002; 25: 547-549.

28. Machi J, Oishi AJ, Furumoto NL et al. Sonographically guided radio frequency thermal ablation for unresectable recurrent tumors in the retroperitoneum and the pelvis. J Ultrasound Med 2003; 22: 507-513.

29. Arellano RS, Flanders VL, Lee SI et al. Imaging-guided percutaneous radiofrequency ablation of retroperitoneal metastatic disease in patients with gynecologic malignancies: clinical experience with eight patients. AJR Am J Roentgenol 2010; 194: 1635-1638.

30. Gao F, Gu Y, Huang J et al. Radiofrequency ablation of retroperitoneal metastatic lymph nodes from hepatocellular carcinoma. Acad Radiol 2012; 19: 1035-1040.

31. Collettini F, Schippers AC, Schnapauff D et al. Percutaneous ablation of lymph node metastases using CT-guided highdose-rate brachytherapy. Br J Radiol 2013; 86: 20130088.

32. Aigner F, Pratschke J, Schmelzle M. Oligometastatic disease in colorectal cancer - how to proceed. Visc Med 2017; 33: 23-28.

33. Wild AT, Yamada Y. Treatment options in oligometastatic disease: stereotactic body radiation therapy - focus on colorectal cancer. Visc Med 2017; 33: 54-61.

34. Pan T, Xie QK, Lv N et al. Percutaneous CT-guided radiofrequency ablation for lymph node oligometastases from hepatocellular carcinoma: a propensity score-matching analysis. Radiology 2016; 282: 259-270. 